\title{
Nachruf auf Prof. Dr. Heinz-Wilhelm Dehne
}

\author{
Erich-Christian Oerke ${ }^{1} \cdot$ Ulrike Steiner $^{1} \cdot$ Richard Sikora $^{1}$
}

Online publiziert: 12. August 2019

(c) Springer-Verlag GmbH Deutschland, ein Teil von Springer Nature 2019

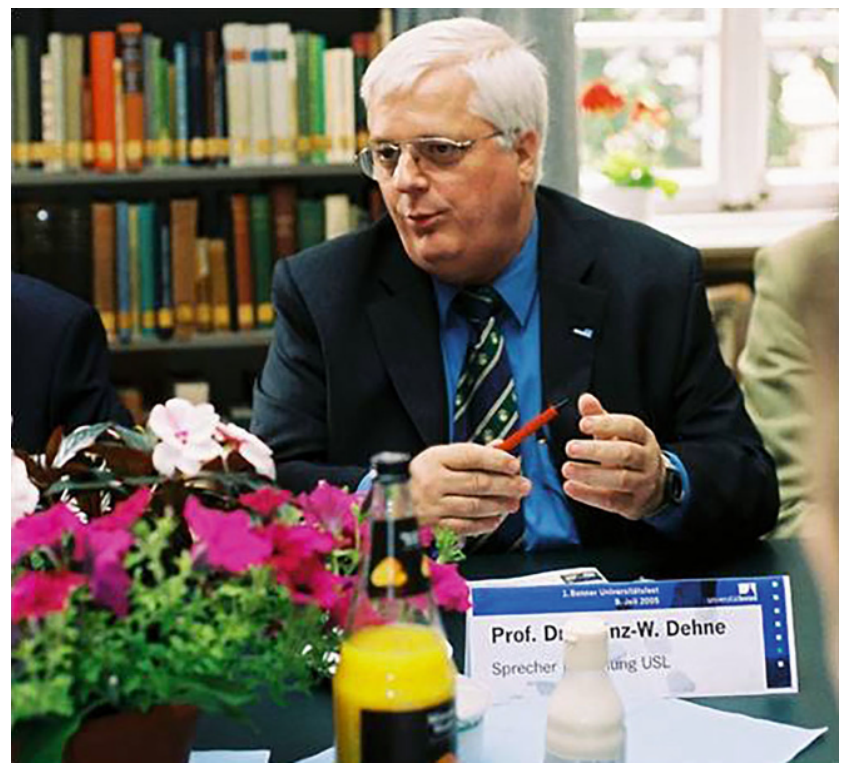

Am 23.05.2019 ist Prof. Dr. Heinz-Wilhelm Dehne nach schwerer Krankheit im Alter von 68 Jahren verstorben.

Heinz-Wilhelm Dehne wurde am 03. August 1950 in Ahnsen/Niedersachsen geboren. Er studierte von 1970 bis 1974 an der Rheinischen Friedrich-Wilhelms-Universität Bonn Agrarwissenschaften. Hier promovierte er auch 1977 bei Prof. Dr. F. Schönbeck mit der Arbeit „Untersuchungen über den Einfluss der endotrophen Mykorrhiza auf die Fusarium-Welke an Tomate und Gurke“. Ab 1975 war er als wissenschaftliche Mitarbeiter, später wissenschaftlicher Assistent, an der Fakultät für Gartenbau der Universität Hannover tätig, wo er 1987 seine wissenschaftlichen Arbeiten in der Habilitationsschrift ,Zur Bedeutung der vesikulärarbuskulären (VA) Mykorrhiza für die Pflanzengesundheit“ zusammenfasste und die Venia Legendi für das Fach Phy-

Erich-Christian Oerke

ec-oerke@uni-bonn.de

1 INRES - Pflanzenkrankheiten und Pflanzenschutz, Rheinische Friedrich-Wilhelms Universität Bonn, Bonn, Deutschland tomedizin erhielt. Anschließend wechselte Heinz-Wilhelm Dehne zur Bayer AG nach Monheim, wo er im Bereich Forschung und Entwicklung von Fungiziden bis zum Frühjahr 1994 tätig war.

Im April 1994 kehrte er an seine Alma Mater zurück. Er übernahm im Institut für Pflanzenkrankheiten der Landwirtschaftlichen Fakultät der Rheinischen Friedrich-WilhelmsUniversität Bonn die Leitung der Abteilung Pflanzenkrankheiten. Nach dem Zusammenschluss der pflanzenbaulichen Institute in das Institut für Pflanzenbauwissenschaften und Ressourcenschutz im Jahr 2006 und dem Ausscheiden von ehemaligen Kollegen wurde die Denomination der Professur um den Begriff Pflanzenschutz ergänzt. Seine Professur hatte Heinz-Wilhelm Dehne bis zu seiner Pensionierung im Februar 2016 inne.

In seiner Zeit als Doktorand und wissenschaftlicher Assistent an der Universität Hannover beschäftigte sich HeinzWilhelm Dehne mit der vesikulär-arbuskulären Mykorrhiza, deren Auswirkungen auf Wachstum und Gesundheit von Pflanzen und mit Nutzungsmöglichkeiten der VAM-Pilze für den praktischen Pflanzenschutz, insbesondere in gartenbaulichen Kulturen. Daneben rückte auch das Konzept der Induzierten Resistenz als Pflanzenschutzverfahren in den Fokus seiner Arbeiten, wobei für ihn immer die Aspekte der Verwendung für den praktischen Pflanzenschutz im Vordergrund standen.

Seine Forschungsschwerpunkte an der Universität Bonn reichten vom Einfluss von Umweltfaktoren auf die Widerstandsfähigkeit von Nutzpflanzen gegenüber Krankheiten an Spross und Wurzel über Strategien zur Vermeidung der Kontamination von Lebens- und Futtermitteln mit Mykotoxinen und die Wirkungsweise von synthetischen und biologischen Pflanzenschutzmitteln bis hin zum Auftreten von invasiven Schaderregern und deren nachhaltiger Bekämpfung. Dabei ist es ihm gelungen, Grundlagenforschung und praktische Pflanzenschutzforschung miteinander $\mathrm{zu}$ verknüpfen und damit erfolgreich zur Lösung drängender Pflanzenschutzprobleme beizutragen.

Heinz-Wilhelm Dehne engagierte sich in verschiedensten Gremien der universitären Selbstverwaltung, u.a. in der Studienkommission Agrarwissenschaften sowie im 
Lehr- und Forschungsschwerpunkt „Umweltverträgliche und Standortgerechte Landwirtschaft". Ein großes Anliegen war ihm der wissenschaftliche Nachwuchs - sowohl in seiner Arbeitsgruppe als auch in der Landwirtschaftlichen Fakultät und in der Deutschen Phytomedizinischen Gesellschaft. Er hat mehr als 50 DoktorandInnen zur Promotion geführt und vier KandidatInnen zur Habilitation begleitet. Seine wissenschaftlichen Leistungen dokumentieren sich in mehr als 90 Publikationen in wissenschaftlichen Zeitschriften sowie zahlreichen Buch- und Tagungsbeiträgen und Artikeln für pflanzenbauliche Fachmagazine. Er war auch an der Konzeptionierung und Erstellung der aktuellen Auflage des Lehrbuchs für Phytomedizin beteiligt.

Er war jahrelang Mitglied der Senatskommission der Deutschen Forschungsgemeinschaft für Stoffe und Ressourcen in der Landwirtschaft sowie Mitglied der Auswahlkommission der Alexander-von-Humboldt-Stiftung sowie Gutachter für nationale und internationale Forschungsträger. In verschiedenen Gremien war er beratend bzw. als Ideengeber konzeptionell tätig. Heinz-Wilhelm Dehne engagierte sich für das Fachgebiet Phytomedizin und Pflanzenschutz in nationalen und internationalen Organisationen, so war er Mitglied u. a. der Deutschen Landwirtschaftsgesellschaft, der Deutschen Phytomedizinischen Gesellschaft und der American Phytopathological Society. In der DPG gehörte er 9 Jahre dem Vorstand an und war von 1996 bis 1999 erster Vorsitzender der Gesellschaft. Für die Jahre 1996 bis 1998 wurde er zum Präsidenten der European Foundation for Plant Pathology gewählt und organisierte das 4. Symposium der EFPP im September 1996 in Bonn. Mitte der 1990er Jahre übernahm er die Organisation des Reinhardsbrunn-Symposiums „Modern Fungicides and Antifungal Compounds" und gestaltete die Veranstaltung bis 2013. Er war darüber hinaus lange Jahre Mitglied des Organisations- und Programmkomitees der Deutschen Pflanzenschutztagung.

Seine wissenschaftlichen Leistungen wurden durch verschiedene wissenschaftliche Auszeichnungen gewürdigt. So wurde Professor Dehne 1998 der Julius-Kühn-Preis der Deutschen Phytomedizinischen Gesellschaft und 2014 in Anerkennung seiner hervorragenden Verdienste um den Pflanzenschutz die Otto-Appel-Denkmünze verliehen.

Seit 2010 litt Heinz-Wilhelm Dehne zunehmend unter gesundheitlichen Problemen. Er ging Ende Februar 2016 in den Ruhestand, wobei er weiterhin noch DoktorandInnen betreute und zur Promotion führte. Letztendlich wurde er von seiner Krankheit besiegt und erlag den Folgen einer schweren Operation. Wir behalten ihn als verdienstvollen Professor und besonderen Menschen in dankbarer Erinnerung. 